\title{
SIMULATING INFRASTRUCTURE OUTAGES: AN OPEN-SOURCE GEOSPATIAL APPROACH
}

\author{
O. Pala ${ }^{1 *}$, P. Schrum ${ }^{2}$ \\ ${ }^{1}$ Center for Geospatial Analytics, North Carolina State University, Raleigh, NC, USA - opala@ncsu.edu \\ ${ }^{2}$ Center for Geospatial Analytics, North Carolina State University, Raleigh, NC, USA - ptschrum@ncsu.edu
}

KEY WORDS: Critical Infrastructure, Utilities, Spatial Decision Support Systems, Cascading Effects, Outage, Undirected Graph, Graph Theory, GIS

\begin{abstract}
:
Understanding the impact of service outages caused by natural or man-made disasters in utility services is a key part of decision-making in response and recovery efforts. Large-scale outages in the last 15 years, from the 2003 northeast blackout to Hurricane Maria devastating Puerto Rico in 2017, highlighted the importance of tight couplings within and across various utilities. The brittleness of these tight couplings results in long delays in restoring large-scale outages. Such cross-infrastructure effects can make analysis for decision makers and responders far more complex. To facilitate recovery, decision makers need to use specialized Decision Support Systems (DSS) that allow simulation of various alternative enablement options along with their impact on society.

In this article, we describe our geo-simulation engine and datasets used for outage modelling. First, we detail our efforts in correcting and completing Electric Power (EP) network for the western US. Next, we explain the architecture and initial implementation of the platform-independent, open-source geospatial simulation engine that we are in the process of developing. Using this engine, we can consider the amount of commodity at the transmission source (power plants) and sinks (substations) and set thresholds at sinks to trigger and simulate outages. For instance, a threshold can be set to trigger an outage at substation level if the available commodity amount drops below $80 \%$ of the demand. Future additions include cross-infrastructure and enablement consequence analysis to provide a complete and transparent DSS to study outages on multiple interrelating infrastructures through scenario-based evaluation criteria.
\end{abstract}

\footnotetext{
$1 *$ Corresponding author
} 


\section{INTRODUCTION AND MOTIVATION}

Decision makers face many challenges in prioritizing resources for protection and response during and after the occurrence of a disaster. In the pre-planning stage, homeland security and emergency management planners discover and isolate weaknesses within a power grid that are likely to be impacted by a disaster. However, this process is often hindered by limited information about the utilities. This is due, in part, to the fact that approximately 85 percent of Critical Infrastructures (CI) in the United States, including power grids, are held by the private sector. These organizations are held responsible for the security of information. Unauthorized access or destruction of an organization's information assets arising from malicious acts, errors, or disasters could result in compromised information, along with numerous other consequences (Holgate et al., 2012).

On the other hand, the growth of information technology has created scenarios in which there is actually an overabundance of data. This scenario creates the necessity for a "needle in the haystack" approach to finding answers in the data. Data quality also factors into the lack of usability of the data risk assessments (Guikema, 2009). As a result, the pre-planning is difficult, may be based on inaccurate assumptions, and may ultimately not be sufficient for quick enactment and remediation during and after a disaster takes place (Havlin et al., 2012).

Magnifying the challenges for analysts and decision-makers are the numerous inherent interdependencies that exist among critical infrastructures (Usov et al., 2010). Modern infrastructures consist of complex cyclic interdependencies. As a result, any restoration process must take a holistic approach to be successful (Coffrin et al., 2012). For example, electric power systems depend upon transportation networks to deliver fuel to generation facilities. These same generation facilities often depend upon water systems for cooling purposes. In addition, electric power systems depend heavily upon telecommunication networks to support the Supervisory, Control and Data Acquisition (SCADA) systems that manage power transmission and distribution (Ebrahimy, 2014).

The list of interdependencies among the CI sectors is long and in many cases, these interdependencies are poorly understood. Furthermore, infrastructure interdependencies are often very strong, time-sensitive, and essential to overarching system operation. The result is a brittle "system of systems" that contributes to the potential for catastrophic occurrences as a failure cascades and escalates across related infrastructures (Havlin et al., 2012). The problem of understanding the behavior of CIs and their interdependence as part of reconstitution efforts remains difficult and open. The limitations of single-dimensional approaches are by no means trivial. Multi-dimensional approaches, while theoretically promising, have produced few results. Analysts and decision-makers face extremely complex issues in understanding and responding to multi-dimensional CI problems. They must account for a variety of contextual elements, including task goals (e.g., remediation vs. response), geographic scope, infrastructure and cross-infrastructure knowledge, resource allocation, and outcome measurement (Usov et al., 2010). Particularly given constraints on response time and dynamic contextual alterations, the complexity can rapidly lead to information overload, which can significantly impact the efficiency and quality of response. In such a shifting decision-making environment, it can be just as important to reduce the decision-making attempt as it is to increase decision quality (Todd et al., 1992). Specifically, simplifying the efforts to prioritize which assets to dedicate resources is vital to recovery following a disaster. This balance is also an important design consideration in CI decision support environments. .

\subsection{Background}

There has been significant interest in analysing interdependencies from various perspectives, mainly resilience, optimization and modelling. In order both to guard against and respond to critical infrastructure failures, multi-dimensional infrastructure modelling and simulation has been proposed as a way to support analysis and decision-making (Rinaldi et al., 2001; Tolone, 2004; Dudenhoeffer, 2006; Santella et al., 2009; Wilson et al., 2009, Eusgeld et al., 2011). In most of these studies, geographic relationships provide a natural foundation for the integration and visualization of multiple infrastructures, scenario scoping and impact analysis.

Studies in the reliability engineering area focusing on interdependence have primarily emphasized topological properties like betweenness and disruption of connectivity (Dueñas-Osorio et al., 2009; Ouyang et al., 2011). Power system restoration scenarios have been considered in studies promoting good methodology in the application of planning, configuration, and diagnostic techniques. However, many researchers use connectivity as a foundation of their models. Hence, their reliability is insufficient in situations in which complex interdependencies exist (Coffrin et al., 2012).

Apostolakis et al., (2005) conducted a study in which they examined three critical infrastructures on the MIT Campus: electric, water and natural gas, as well as the interactions between them. They focused on developing a process for identifying critical locations in infrastructures, given a specific threat as a component of their vulnerability analysis. Following on that work, Michaud et al., (2006) developed an approach using geographical information that takes water supply network capacities and repair into account to create a vulnerability screening methodology in the context of potential terrorism scenarios. Patterson et al., (2007) pooled the prior two methods and tailored them to produce a more effective system for mapping the geographic-valued worth, which was exhibited in a color scheme representative of the numerical ranking of distinct geographical areas. Koonce et al., (2008) performed bulk power risk analysis by calculating risk significance of each infrastructure element and ranking them.

Tolone et al., (2010) have created a multi-infrastructure modeling system that can simulate interdependencies and vulnerabilities among numerous entities. Through simulation, the impacts of infrastructure failure components on other infrastructures can be predicted. However, this system requires substantial collaboration and data sharing.

Coffrin, et al (2012) devised a "last-mile restoration approach" to be applied to multiple complex interdependent infrastructures. This approach uses mixed-integer programs to model interdependent networks (specifically, power and gas), through the combination of a linearized Direct Current model for the first network (power) and a flow model for the other. Usov et al., (2010) recommends simulation for critical infrastructure dependency analysis to test methods of risk reduction, and evaluation of historical failures. Moreover, coupling the simulations with external threat models, such as a river flood model, can help with decision-making processes in more complex situations. 
Mendonça, et al. (2014) published a description of a group of prototype tools that were designed for analysis in post-disaster environments and supporting the restoration of infrastructure systems through training exercises. The system consists of largescale displays, novel interaction abilities, and realistic data, all run through discrete event simulations. The parameters (i.e. time available for task execution, the complexity of the networks) of each simulation could be modified as needed. This work shows promise for aiding in understanding complex interactions within and among infrastructures.

Kulawiak et al., (2013) presented a system offering tools for target analysis, simulations, and spatial analysis for use in analyzing municipal Critical Infrastructures with a remote, webbased geographic information system. This system was applied to research in the city of Gdansk, Poland, including blast attack, chemical contamination, and flood hazard scenarios. The system also used a spatial density algorithm, that identifies events where the proximity of certain infrastructures can influence their susceptibility to attack. Liu (2014) used a computational model of failures (incorporating field knowledge, records, results from inspection, and sensory data) within the infrastructure of water transmission and distribution systems, with the goal of facilitating the decision-making process in water main renewal.

Pala, et al. (2013) implemented a "proof of concept" decision recommendation system for a university campus and showed the effectiveness of a geovisualziation recommendation approach through a user study testing. More recently, Portante, et al. (2017) implemented a fully functional system that works with power and NG networks simultaneously. Even though this system shows promise, it is developed by US Department of Energy and therefore not readily available to research community. In addition, it requires detailed technical data on each network to function and detailed information about inner workings of the system is not available.

Each of the above mentioned studies has contributed to the general understanding of interdependencies among infrastructures. Ouyang (2014) reviewed studies across the critical infrastructure field and broadly groups the existing modeling and simulation approaches into six types: empirical approaches, agent based approaches, system dynamics based approaches, economic theory based approaches, network based approaches, and others. The author also offers future research directions and identifies critical challenges in the field. Future directions that are identified are a) "data access and collection," b) "comprehensive modeling and analysis," c) "Integration and co-simulation" and d) "validation and applications".

Our research is an initial step towards addressing Ouyang's areas of comprehensive modeling and analysis, validation, and integration. Here, we present a description of our recent and ongoing work to progress towards improved understanding of the nature of cross-network disablements and to allow end-user simulation of faulting and repairing the networks. Specifically we are at the beginning stages developing a Spatial Decision Support System (SDSS), which will have the capability of modelling multiple interdependent CI networks at the transmission level while displaying the network component locations and service states. In the next section, we will explain the data structure, our efforts in data synthesis through geospatial conflation of various power network data, and creation of a system that ingests geospatial network data to simulate outages to meet a specific level of demand.

\section{METHODOLOGY}

In this research, we focus on planning and decision-making efforts in the aftermath of a disaster. We have developed an innovative approach to modelling critical infrastructures in order to support decision-making during reconstitution efforts in response to infrastructure disruptions.

Utility entities of different types are required by US federal government to keep their databases confidential and secure to protect from information-based attacks, whether in the cyber or physical domains. Hence, information sharing is strictly reduced. Yet utilities of various commodity types depend on each other to function adequately. Because of this interdependency, a failure on one type of network can fail over onto another network, causing cascading failures traversing type boundaries. The secure nature of the information held by the different entities becomes a liability during failures in that it blocks the dissemination of system state knowledge among decision makers needed for them to have a full understanding of their current outage scenario.

\subsection{Data}

Geospatial data representing CIs are usually not publicly available and it is often difficult to find open data sources. Available data is highly generalized and lacks technical detail that would be required to perform meaningful modelling for simulation purposes. This is due to the highly sensitive nature of the information. Hence, our goal in using this data is not to represent a perfect model of the world, but to reconstruct a reasonable synthesis of plausible data topology. Initially our development effort has focused on modelling the Power Transmission Network. Modelling of other kinds of networks such as Natural Gas (NG) is planned for near future.

Homeland Security Infrastructure Program (HSIP, 2016) provides data to US federal agencies. HSIP Gold, unclassified on in 2015, is a unified homeland infrastructure geospatial data inventory assembled by National Geospatial Agency in partnership with the Department of Homeland Security. It is a compilation of over 560 geospatial datasets, characterizing domestic infrastructure. HSIP geospatial data was later named HIFLD (2015) and more recently is made available through federal geospatial portal.

The dataset we used was a generalized form of HSIP 2013 data. We obtained this dataset on ArcGIS online data portal (2015). It consists of two feature classes, one for power transmission and the other for power nodes, which are either sources (power plants) or sinks (substations, which step voltage down and supply distribution networks).

\subsection{Data Pre-Processing}

When we obtained the initial dataset, it was not ready to be used in creating the network model. Specifically, it did not distinguish sink or source nodes, nor did it have source power production capacities. Also it did not include nodes at network tees, and node-to-edge connectivity did not exist.

The model requires there to be nodes at Tees, so we manually added those using ArcGIS 10.x tools, which created a new feature class for nodes. We then obtained a comprehensive list of power plants including coordinates, capacity and fuel type on an information repository website (Wikipedia, 2016). This data was converted to csv file, and then brought into ArcMap to create a new feature class of power plants. The coordinates for the power 
plants created from information repository website do not exactly match the coordinates for nodes downloaded from HIFLD (2016). Therefore each power plant had to be associated with a node using the "nearest" processing tool from this power capacity and fuel type was transferred into the node after you table.

The final pre-processing step is to add a column to the nodes attribute table which represents edge connectivity. This is accomplished using a Python tool that creates a pipe delimited string of all edge IDs that connect to each individual node. When the model is being loaded, the list of edges connected to the given node is generated from the pipe limited list. This enables the network model to be an oriented undirected graph. Once data preprocessing is complete, it does not need to be repeated for any given dataset.

\subsection{Network Modelling and Software Development}

After the data has been prepared by pre-processing, the main modelling tool is used. The functionality of this is separated into two modules, CIModellerBase (Base) and CIModellerGUI (GUI). Network models and associated functionality are embodied in CIModellerBase that has no external build dependencies other than the .NET framework. Persistence, visualization, and Graphical User Interface are provided via ArcGIS Pro (Pro) through CIModellerGUI, which is an ArcGIS Pro add-in. It also has a dependence on CIModellerGUI. Figure 1 illustrates this build dependency relationship.

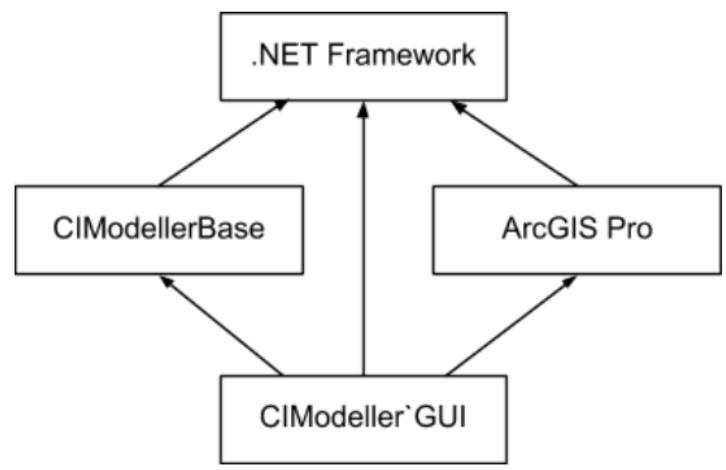

Figure 1. Build Dependency Diagram showing build dependencies among modules and ArcGIS Pro

Isolating graph (network) functionality in Base achieves loose coupling, so it may be built, tested, and called without the other components. For use within a GIS (ArcGIS Pro in this case), CIModellerGUI serves to bridge ArcGIS Pro and CIModellerBase. Isolating CIModellerBase's dependencies means that the module may be used by other Geographic Information Systems such as GRASS GIS or QGIS. In that case, programmers would develop their own bridge module in place of CIModellerGUI.

When executing unit tests, CIModeller supplies its own data reading function which reads network data from csv files. When executing the ArcGIS Critical Infrastructure add-in, CIModellerGUI provides a callback function to CIModellerBase via an interface which passes reading and writing of shapefile attribute tables up to ArcGIS Pro API functions. Differing states of network components are displayed in different symbology using ArcGIS Pro's symbology capabilities combined with filtering network elements via Definition Queries. Table 1 summarizes which components of Figure 3 are responsible for which responsibilities.

\begin{tabular}{|l|l|}
\hline Component & Responsibilities \\
\hline CIModellerBase & $\begin{array}{l}\text { - Model the network Graph } \\
\text { Implement Faults, Repairs } \\
\text { - Compute connected } \\
\text { components } \\
\text { - Compute commodity } \\
\text { supply states for each } \\
\text { connected component }\end{array}$ \\
\hline CIModellerGUI & $\begin{array}{l}\text { Implement AddIn } \\
\text { functionality, including } \\
\text { button code } \\
\text { - Hold instance of Network } \\
\text { Universe and call it } \\
\bullet \text { Host Attribute Table Read } \\
\text { and Write Functionality }\end{array}$ \\
\hline ArcGIS Pro & $\begin{array}{l}\text { - Save Attribute Data } \\
\text { - Visualize Map } \\
\bullet \text { Host the AddIn }\end{array}$ \\
\hline
\end{tabular}

Table 1: Summary of Software Components and their process responsibilities

As shown in the above table, CIModellerBase is the graph engine for the system, and GUI serves as an adapter between Base and the GIS, which is ArcGIS Pro in this case.

\subsection{The Network Model}

Setola et. al., (2016), note that graph theory may be applied to model network functionality. The present model follows this pattern in that all network functionality is implemented in "Base" module. Each network is modelled as an undirected, oriented graph. Graph nodes represent commodity sources, sinks, or tees. Graph edges represent physical commodity traversal conduits, i.e., power transmission lines or gas or water pipelines. Networks are modelled as a collection of nodes and a collection of edges. Each node holds a collection of edges which connect to it. Each edge holds members Left Node and Right Node. Commodity may flow either direction, so the graph is undirected. To model flow, its direction must be known, so the graph is oriented.

\subsection{Object Orientation}

To maximize code reuse and to exploit other benefits of object oriented programming, nodes and edges exist in the same inheritance hierarchy, as shown in Figure 2.

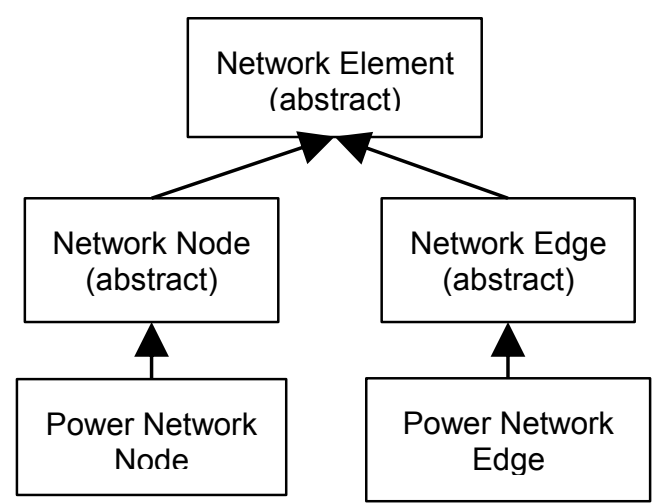

Figure 2: Inheritance Hierarchy of Network Nodes and Edges

Most network functionality is embodied in Network Node and Network Edge. Certain functionality essentially depends on the type of commodity being conveyed. For example, the essential 
computation of flow resistance is different for power lines than it is for water pipes. In order to allow for this difference, different concrete classes are required for power network edges and water network edges. This is to prepare for when the transfer capacity based flow modelling is implemented.

\subsubsection{Model Object Hierarchy:}

In order to model cross-network failover cascade events, all networks must be contained in the same entity. To accomplish this, all networks are contained inside a single, top-level object of type Network Universe. To account for the possibility that the various network datasets contain more than one distinct network, disconnected networks of the same type are kept in an object of type Network Group. Specific commodity types (such as Power Network Group) inherit from Network Group. Each Network Group keeps a collection of individual Commodity Networks. Each Commodity Network keeps a collection of Nodes and Edges. This object hierarchy is depicted in Figure 3. All components of this model are implemented in Base.

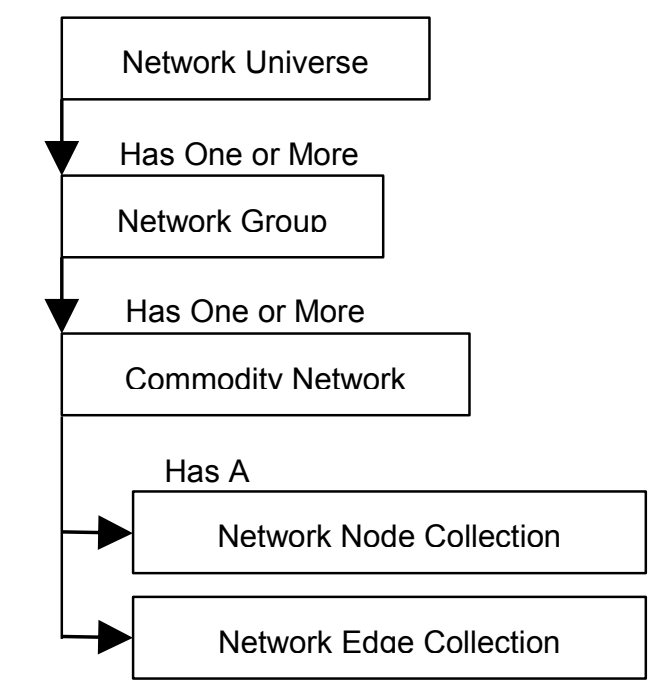

Figure 3: Model Object Hierarchy for CIModellerBase

\subsection{The ArcGIS Pro AddIn, CIModellerGUI}

To interact with the network model through ArcGIS Pro, there must be a C\# module which inherits from ArcGIS.Desktop.Framework.Contracts.Module. If the design involved CIModellerBase inheriting from Module, build independence would be forfeited and any user would be required to have an ArcGIS Pro license to run it. Thus we introduced the intermediate module, CIModellerGUI (GUI). GUI inherits from Module and serves as the ArcGIS Pro AddIn, Critical Infrastructure.

\subsection{Callback Functionality}

Once Base has completed a graph operation, and connected components have been computed, the new state of the network universe must be propagated back to the GIS, ArcGIS Pro in our case. Because Base has no external dependencies, it does not "know" how to save values associated with the newly computed state.
In order to accomplish the saving of the new state values, GUI, which has a function which persists values to the proper Attribute Tables through the ArcGIS Pro API. For Base to be able to call this function, GUI provides Base with the function reference, which Base calls after making computations.

All buttons which appear on the Critical Infrastructure tab of ArcGIS Pro are implemented in GUI in Esri's Desktop Application Markup Language (DAML). Button "code behind" functions are functions in GUI, and primarily serve to call functions residing in Base which perform actual Graph computations.

The Graph module, implemented by Base, is instantiated in GUI when a user selects the "Load Networks" button on the Critical Infrastructure tab of the AddIn. The entire model is held by GUI as a single instance of class NetworkUniverse. All functionality can be accessed via this reference.

\subsection{Connected Component Determination and Powered State}

Each network is modelled as an oriented undirected graph. $\mathrm{G}=$ $(\mathrm{N}, \mathrm{E})$, in which $\mathrm{N}$ is a collection of nodes and $\mathrm{E}$ is a collection of edges. Each edge is connected to two nodes. Each node is connected to one or more Edges. When the network universe is first loaded, connected nodes and edges are traversed to determine the extent of one or more distinct networks. At this initial stage, each network has only a single connected component, itself.

Steps 6 and 7 of the Internal Communication Trace (below) are key aspects of the graph modelling taking place in Base. When a fault isolates one part of a network from the rest of the network, the result is two or more connected components. All elements in the faulted state are also false for their "conveys power" attribute. This leads a disconnection in the graph, resulting in its bifurcation into multiple connected components.

After all connected components have been identified each one has its total available power computed. For each connected component, sink power demand is totalled, as is source power supply. These two are added to result in residual connected component power supply. If there are no sources, all sinks are at $0 \%$ and are identified as unpowered. If the total available power divided by the total sink demand is less than $100 \%$, all sinks are identified as underpowered.

Let $\mathrm{N}_{\text {source }} \subseteq \mathrm{N}$ denote the collection of nodes of $\mathrm{G}$ that represent power sources, and let $\mathrm{N}_{\text {sink }} \subseteq \mathrm{N}$ denote the collection of nodes of $\mathrm{G}$ that represent sinks. The power supply at $\mathrm{x} \in \mathrm{N}_{\text {source }}$ is denoted $\mathrm{s}\left(\mathrm{x}_{\mathrm{i}}\right)$, and the power demand at $\mathrm{x} \in \mathrm{N}_{\text {sink }}$ is denoted $\mathrm{d}\left(\mathrm{x}_{\mathrm{i}}\right)$. The total available power supply of the network is given by

$$
\mathrm{S}=\Sigma \mathrm{s}\left(\mathrm{x}_{\mathrm{i}}\right) \quad \text { over all } \mathrm{xi}_{\mathrm{i}} \in \mathrm{N}_{\text {source }}
$$

The total demand of the network is given by

$$
\mathrm{D}=\Sigma \mathrm{d}\left(\mathrm{x}_{\mathrm{i}}\right) \quad \text { over all } \mathrm{x}_{\mathrm{i}} \in \mathrm{N}_{\text {sink }}
$$


When a fault takes place on the edge e in $\mathrm{G}$, the attribute isFaulted is changed to true. Let $\mathrm{G}^{\prime}$ be the "non-faulted" subgraph (N, E $\{\mathrm{e}\}) \subseteq \mathrm{G}$ where the edge set consists of edges which are not faulted. This subgraph of $\mathrm{G}$ may contain multiple connected components $\mathrm{H}_{1}, \mathrm{H}_{2}, \ldots, \mathrm{H}_{\mathrm{n}}$. We identify each of these components. For each component $\mathrm{H}_{\mathrm{j}}$ we compute the total power $\mathrm{S}_{\mathrm{j}}$, the total demand $\mathrm{D}_{\mathrm{j}}$, and the LoadPercent $\mathrm{L}_{\mathrm{j}}$.

$$
\begin{aligned}
& S_{j}=\Sigma s\left(x_{i}\right) \quad \text { over all } x_{i} \in H_{j} \cap N_{\text {source }} \\
& D_{j}=\Sigma d(x i) \quad \text { over all } x i \in H j \cap \text { Nsink } \\
& L_{j}=\left(S_{j} / D_{j}\right) * 100
\end{aligned}
$$

For every connected component $\mathrm{j}$, if $\mathrm{L}_{\mathrm{j}}$ is $0, \mathrm{H}_{\mathrm{j}}$ is unpowered. If $\mathrm{L}_{\mathrm{j}}$ is between 1 and 99 , it is underpowered. If $\mathrm{L}_{\mathrm{j}}$ is 100 or more, then $\mathrm{H}_{\mathrm{j}}$ is fully powered. Over-powered situations are currently treated as fully powered.

\subsection{End User Workflow}

The geovisualization part of the application runs as an add-in to ArcGIS Pro (v 2.0). As a part of the interactive user interface we have implemented an Critical Infrastructure ribbon as an add-in (Figure 1).

The end user begins a session by clicking the "Load Networks" button. In order to simulate a system fault, the user selects one or more network items in the Map window, then implements the fault in the model by clicking the "Fault Selected Elements" button. The add-in computes the new state of the network based on the newly faulted elements. It saves the new state in the memory cache of ArcGIS Pro, and shows under-powered and unpowered nodes with symbology to visualize the impaired power state.

In order to repair one or more faulted elements, the end user selects a faulted element on the map and clicks the "Repair Selected Elements" button. The add-in computes the new state of the network based on the new combination of faulted elements. It saves the new state in the memory cache and again shows under-powered and unpowered nodes (if any) with symbology to visualize state.

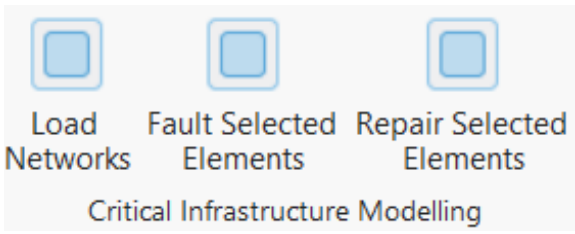

Figure 4: Critical Infrastructure Tab of the ArcGIS Pro Add-In

By using ArcGIS Pro, persistence in a feature class attribute table and visualization on a map are provided without additional software development. Due to Pro's internal persistence model, persistence takes place first in a temporary cache, which is what is displayed on the map. This means that changes are not saved to long term persistence (i.e., on the hard drive) until requested by the end user.

The workflow for the end user is simple (Figure 4):

After loading the model by clicking the Load Networks button:

1. The user selects one or more network elements on the map.

2. The user clicks either the Fault Selected Elements button or the Repair Selected Elements button.
Upon performing Step 2, all connected components are recomputed and the new state of the entire Network Universe is displayed on the map. Faulted items are show in red. Unpowered and underpowered elements are displayed with black or grey " $\mathrm{X}$ " icons. See the Results section for screen shots of these.

\subsection{Internal Communications Trace}

The simplicity of the above workflow is accomplished internally by multi-step internal communication among the components depicted in Figure 2, separated into these responsibilities enumerated in Table 1:

1. Pro accepts the button click and passes control to GUI.

2. GUI gets a collection of selected items from Pro.

3. GUI filters items not part of a loaded network from the collection.

4. GUI tells Base which items are faulted.

5. GUI directs Base to recompute all connected components on affected networks

6. Base recomputes connected components.

7. For each connected component, Base recomputes powered state then applies its powered state to each of its sinks.

8. GUI writes new powered status to the Attribute Table.

9. GUI directs Pro to clear selected elements.

10. GUI returns control to Pro.

11. Pro detects changes to the Attribute Tables and updates the Map display.

The system performs this operation on the Western United States power grid dataset. We will explain the results of such outage simulations in the Results section below.

\section{Results}

Several trial simulations were carried out to confirm that the expected behavior takes place. Figure 5 shows the impact of the faulting of a section of a power transmission network in which a faulted transmission line (in red) has resulted in unpowered 6 sinks (substations, shown with black X-marks).

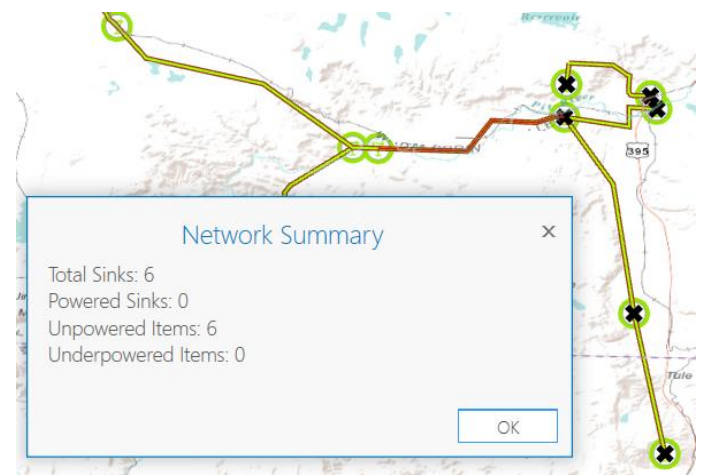

Figure 5: Scenario 1, A simulated fault and resulting outage on a section of a power transmission network using the Critical Infrastructure add-in to ArcGIS Pro

The results are presented graphically as part of the map, and also textually in the message box. More comprehensive and informative textual information will be developed in the future.

Figure 6 shows a second scenario, a fault on five transmission lines results in a large area becoming unpowered. 


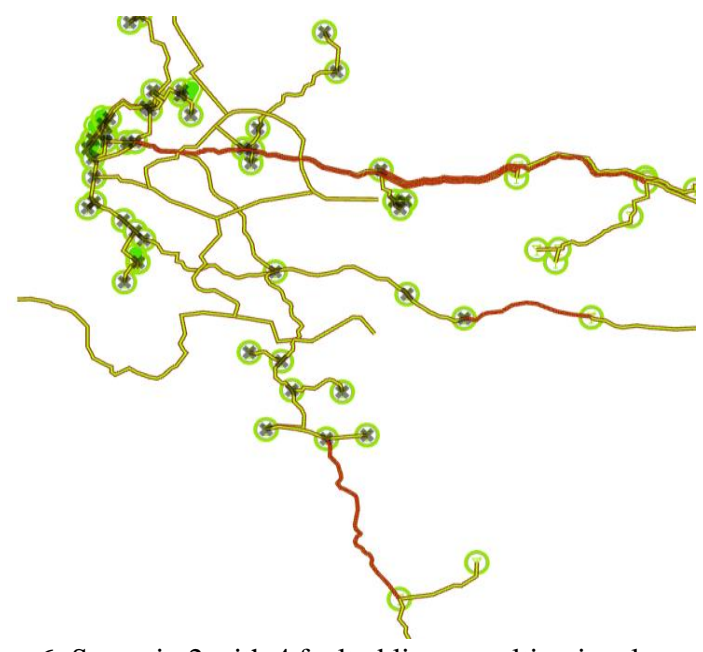

Figure 6: Scenario 2 with 4 faulted lines resulting in a large area outage.

The end user continues with this same exercise by repairing one of the faulted lines. The user selects a faulted item, ID 1171 in this case, indicated by the dashed line crossing it. With this item selected, the user selects the Repair Selected Items button, and the resulting state of the network is shown in Figure 7.

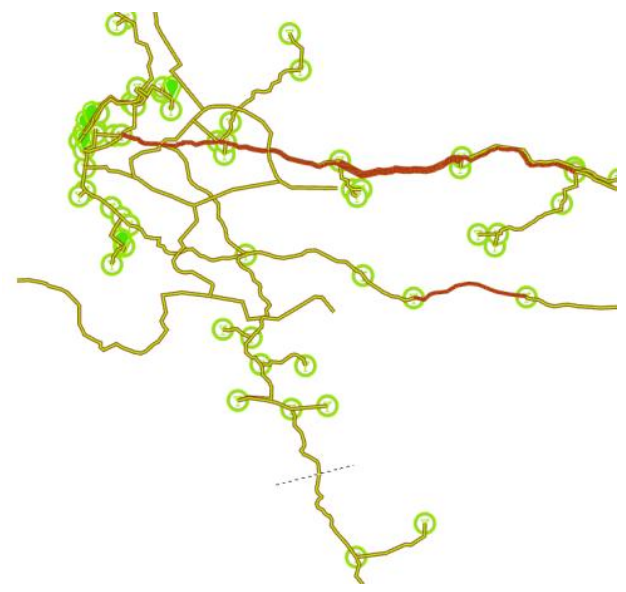

Figure 7: Scenario 2A in which repairing just one line results in all sinks being powered

Because the other lines are still faulted, all of the simulated power supplied to these sinks comes through this one line, ID 1171. In a real world situation like this, it is possible that the single transmission line of ID 1171 may not have enough capacity to carry all of this power, and the line may fault itself as a result Modelling this type of scenario will require flow modelling, which will be implemented in future work.

\section{COnClusion And Future Work}

The decision-making environment both during and after an infrastructure disaster can be extremely complex and dynamic. Decision makers may need to re-evaluate plans rapidly in response to changing conditions. For example, the environment during a terrorist attack can be considered as opposing sides wanting to control the states of the critical infrastructure systems in order to achieve their goals (Haimes 2006). In such a changing decision-making environment, reducing the decision-making effort can be as important as increasing the decision quality (Todd et. al., 1992).
The work we have reported here is a first step in creating a complex system that analyses multiple critical infrastructure networks and employs multiple ranking factors in order to provide reconstitution support for the decision maker.

The system we are presenting here is based on a self-contained code base that we are developing from the ground-up. It only relies on a geospatial platform for the user interaction portion, to export the network data and to show results in the form of interactive geosimulation. Even though we have implemented our solution with a commercial GIS package (ArcGIS Pro), our solution is not based on a specific geospatial software package. It is a geospatial platform independent code-base which could be implemented on commercial or open-source GIS platform with minimal effort. In order to implement this, we obtained a power network dataset for a large portion of the western US and preprocessed this data to match nodes to ends of lines nearby within a threshold of distance. Here nodes are either power sources (i.e. power plants and other power generation facilities), taps, or power sinks (i.e. substations of various sizes and other power conversion locations). Lines are power transmission lines of various voltages. We currently are able to simulate an outage and set a threshold for minimal demand to be met before every node on a subgraph is disabled. We designed the system to be easily extendible to include other networks such as natural gas (NG) and water with small modifications to the existing codebase.

The next step in this research is to add the ability to ingest NG network and to set up the system to simulate an outage on the NG network. Adding the cross-infrastructure effect simulation will allow us to set up scenario-based cascading outage simulations across multiple infrastructure networks and provide enablement options to decision makers. These options will include a decision table and each network in a tree view and effect of the outage on each branch with an outage on a specific targets such as population or medical facilities or financial institutions.

We also plan to study the decision process for selecting the best outcome when there are multiple decision criteria with spatial components. Part of this would be evaluating which criteria are more important in which type of disasters. For example, bringing up the water network first would make sense if there is a disaster involving severe fire hazards but it might be more beneficial to bring the power network up if there is a winter storm so people can warm up their houses. In addition, we would have to decide on which targets are more important in which kind of disasters. For example, providing power and water to hospitals could gain priority in a disaster where there are lots of causalities. This would mean that the target entity would be hospitals and the goal would be to maximize the number of hospitals that are functional. Whereas providing power to the most number of people might be more logical in a disaster with no casualties but a lot of damage to property. These examples open the possibility of actually assigning different values to different target entities and infrastructures in different types of disaster. This would allow us to study the selection of best possible point of enablement as a multi-criteria and multi-attribute decision making process.

\section{ACKNOWLEDGEMENTS}

We thank Dan Scofield, Ph.D. candidate in Mathematics at North Carolina State University for his help with graph notation and equations. 


\section{REFERENCES}

Apostolakis, G.E., Lemon, D.M., 2005. A screening methodology for the identification and ranking of infrastructure vulnerabilities due to terrorism. Risk Analysis 25, 361-376.

ArcGIS Data Portal (2015): http://www.esri.com/arcgisonline last access 11.10 .2015

Coffrin, C., Van Hentenryck, P., \& Bent, R. (2012). Last-Mile Restoration for Multiple Interdependent Infrastructures. In Proceedings of the AAAI Conference on Artificial Intelligence.

Dueñas-Osorio, L., Vemuru, S.M., 2009. Cascading failures in complex infrastructure systems. Structural safety 31, 157-167.

Dudenhoeffer, D.D., Permann, M.R., Manic, M. 2006. CIMS: a framework for infrastructure interdependency modeling and analysis, Proceedings of Winter Simulation Conference.

Ebrahimy, R. (2014). Investigating SCADA Failures in Interdependent Critical Infrastructure Systems. arXiv preprint arXiv: 1404.7565 .

Eusgeld, I., Nan, C., Dietz, S., 2011. "System-of-systems" approach for interdependent critical infrastructures. Reliability Engineering \& System Safety 96 (6), 679-686.

Guikema, S.D., 2009. Natural disaster risk analysis for critical infrastructure systems: An approach based on statistical learning theory. Reliability Engineering \& System Safety 94, 855-860.

Haimes, Y.Y., 2006. On the definition of vulnerabilities in measuring risks to infrastructures. Risk Analysis 26, 293-296.

Havlin, S., Kenett, D., Ben-Jacob, E., Bunde, A., Cohen, R., Hermann, H., Kantelhardt, J., Kertész, J., Kirkpatrick, S., Kurths, J., 2012. Challenges in network science: Applications to infrastructures, climate, social systems and economics. European Physical Journal-Special Topics 214, 273.

HIFLD Homeland Infrastructure Foundation-Level Data (2015): https://hifld-geoplatform.opendata.arcgis.com/

Holgate, J., Williams, S.P., Hardy, C.A., 2012. Information Security Governance: Investigating Diversity in Critical Infrastructure Organizations. Bled eConference, p. 13.

HSIP Homeland Security Infrastructure Program (2016): https://gii.dhs.gov/HIFLD/hsip-guest

Koonce, A.M., Apostolakis, G., Cook, B., 2008. Bulk power risk analysis: ranking infrastructure elements according to their risk significance. International Journal of Electrical Power \& Energy Systems 30, 169-183.

Kulawiak, M., Lubniewski, Z., 2013. SafeCity-A GIS-based tool profiled for supporting decision making in urban development and infrastructure protection. Technological Forecasting and Social Change 89, 174-187.

Liu, Z., Kleiner, Y., 2014. Computational Intelligence for Urban Infrastructure Condition Assessment: Water Transmission and Distribution Systems. IEEE Sensors Journal 14, 4122-4133.
Mendonça, D., Cutler, B., Wallace, W.A., Brooks, J.D., 2014. Collaborative Training Tools for Emergency Restoration of Critical Infrastructure Systems, ADV INTELL SYST, Volume 1. Springer, pp. 571-581.

Michaud, D., Apostolakis, G.E., 2006. Methodology for ranking the elements of water-supply networks. Journal of Infrastructure Systems 12, 230-242.

Ouyang, M., Dueñas-Osorio, L., 2011. An approach to design interface topologies across interdependent urban infrastructure systems. Reliability Engineering \& System Safety 96, 1462-1473.

Pala, O., Wilson, D.C., 2013. User Study Analysis of a Geovisualization Decision Support Environment for Critical Infrastructure Recovery, Association of Geographic Information Laboratories in Europe (AGILE) 2013, Leuven, Belgium.

Patterson, S.A., Apostolakis, G.E., 2007. Identification of critical locations across multiple infrastructures for terrorist actions. Reliability Engineering \& System Safety 92, 1183-1203.

Portante, E.C., Kavicky, J.A., Craig, B.A., Talaber, L.E., Folga, S.M., 2017. Modeling Electric Power and Natural Gas System Interdependencies. Journal of Infrastructure Systems 23 (4), 04017035 .

Santella, N., Steinberg, L.J., Parks, K., 2009. Decision Making for Extreme Events: Modeling Critical Infrastructure Interdependencies to Aid Mitigation and Response Planning. Review of Policy Research 26 (4), 409-422.

Setoloa, R., Vittorio, R. Kyriakides, E., Rome, E, editors, Managing the Complexity of Critical Infrastructures: A Modelling and Simulation Approach, Springer International Publishing, Cham, Switzerland, p 98.

Todd, P., Benbasat, I., 1992. The use of information in decision making: an experimental investigation of the impact of computer-based decision aids. MIS Quarterly, 373-393.

Tolone, W.J., Wilson, D.C., Raja, A., Xiang, W.-n., Hao, H., Phelps, S., Johnson, E.W., 2004. Critical infrastructure integration modeling and simulation. Intelligence and Security Informatics. Springer, pp. 214-225.

Tolone, W.J., Chu, B.-t., 2010. Multi-infrastructure modeling system. Google Patents.

Usov, A., Beyel, C., Rome, E., Beyer, U., Castorini, E., Palazzari, P., Tofani, A., 2010. The DIESIS approach to semantically interoperable federated critical infrastructure simulation, Second International Conference on Advances in System Simulation (SIMUL). IEEE, pp. 121-128.

Wikipedia (2016), various authors, List of power stations in California,

https://en.wikipedia.org/wiki/List_of_power_stations_in_Califo rnia (23 October 2016)

Wilson, D.C., Pala, O., Tolone, W.J., Xiang, W.-N., 2009. Recommendation-based geovisualization support for reconstitution in critical infrastructure protection, Proceedings of SPIE 7346, Visual Analytics for Homeland Defense and Security Orlando, FL, pp. 734602-734602. 